\title{
A proposta da Redescrição Representacional como referencial para a conceitualização de modelos na educação científica
}

\section{Representational Redescription as a framework for model conceptualization in Science Education}

\author{
Juliana Machado ${ }^{1}$ \\ https://orcid.org/0000-0002-1972-7854 \\ Marco Braga ${ }^{2}$ \\ https://orcid.org/0000-0002-1289-9178
}

\begin{abstract}
Resumo: Apresentamos e discutimos a proposta da Redescrição Representacional, teoria cognitiva desenvolvida pela psicóloga Annette Karmiloff-Smith que oferece um quadro explicativo sobre como a mente humana explora e organiza o conhecimento de tal modo a possibilitar um autoenriquecimento. Nessa proposta, o desenvolvimento de teorias-em-ação por parte do sujeito cognitivo é compreendido em termos de um processo reiterativo de reelaboração de suas representações internas, o qual nem sempre envolve a tomada de novas informações do ambiente externo. Neste artigo, após traçar um breve panorama dos estudos sobre a modelização do ponto de vista cognitivo, discutimos algumas possíveis contribuições da proposta da Redescrição Representacional para a compreensão do processo de conceitualização de modelos na Educação Científica, particularmente em relação ao papel atribuído por Karmiloff-Smith aos conhecimentos implícitos, à sobregeneralização e à flexibilidade cognitiva.
\end{abstract}

Palavras-chave: Redescrição representacional. Desenvolvimento cognitivo. Construção de modelos. Educação em ciências.

\begin{abstract}
We present and discuss the proposal of Representational Redescription, a cognitive theory developed by psychologist Annette Karmiloff-Smith, which offers an explanatory framework on how the human mind explores and organizes knowledge in order to enable self-enrichment to take place. In this proposal, the development of theories-in-action by the cognitive subject is understood in terms of a reiterative process of re-elaboration of internal representations. This process does not always involve the taking in of new information from the external environment. After a brief overview of the studies on modeling from a cognitive viewpoint, we discuss some possible contributions of the Representational Redescription proposal for understanding the process of model conceptualization in Science Education, particularly in relation to the role assigned by Karmiloff-Smith to implicit knowledge, overgeneralization and cognitive flexibility.
\end{abstract}

Keywords: Representational redescription. Cognitive development. Model construction. Science education.

\footnotetext{
${ }^{1}$ Centro Federal de Educação Tecnológica Celso Suckow da Fonseca (CEFET), Programa de Pós-Graduação em Ciência, Tecnologia e Educação, Rio de Janeiro, RJ, Brasil. E-mail: juliana.machado@cefet-rj.br

${ }^{2}$ CEFET, Diretoria de Pesquisa e Pós-graduação (DIPPG), Rio de Janeiro, RJ, Brasil.
} 


\section{Introdução}

A importância atribuída aos temas modelos e modelização para a educação científica, principalmente a partir dos anos 2000, é tal que muitos autores consideram a habilidade de usar, construir e compreender modelos como central para comprender a própria ciência. Assim, por exemplo, Harrison e Treagust $(1998,2000)$ destacam que a construção de modelos constitui a essência do pensamento e do trabalho científico. Para Coll, France e Taylor (2005), os modelos são uma ferramenta-chave para cientistas, professores e estudantes de ciências e o papel crucial deles na Ciência justifica sua inserção no ensino.

Ao mesmo tempo, pesquisas na área de ensino de ciências têm revelado que os estudantes tendem a compreender modelos mais como cópias da realidade do que como representações conceituais, parciais e aproximativas (COLL; FRANCE; TAYLOS, 2005; HARRISON; TREAGUST, 2000; MACHADO; BRAGA, 2018; TABER, 2012). Essa constatação parece indicar a existência de alguma dificuldade em distinguir entre os objetos da investigação científica e os conhecimentos científicos produzidos sobre esses objetos. De um ponto de vista epistemológico, essa problemática se insere na relação entre a realidade e as teorias científicas que versam sobre essa realidade. Nessa relação é que diversos autores - tanto do campo da filosofia da ciência quanto da área da educação científica - têm apontado a relevância dos modelos como elementos mediadores (GILBERT et al., 2000).

Modelos tais como espécies, células, átomos, raios luminosos, massas pontuais, corpos rígidos e redes cristalinas encontram-se tão presentes no ensino de ciências quanto no próprio arcabouço da ciência, dado que não é possível uma apropriação de conhecimento científico - seja pelo especialista, seja pelo estudante - na ausência de representações conceituais, cujo processo de elaboração envolve abstrações e idealizações. Essa ubiquidade dos modelos é o que conduz autores como Gilbert e Justi (2016) a destacarem que uma aprendizagem autêntica na educação científica envolve necessariamente um ensino baseado em modelização. Nessa ótica, uma questão relevante e complexa é: como os estudantes conceitualizam os modelos científicos escolares? Sem pretender esgotar um tema tão vasto, nas páginas que seguem propomos uma discussão do problema a partir de um ponto de vista cognitivo. Nela, resgatamos e discutimos possíveis contribuições do referencial teórico da psicóloga Annette Karmiloff-Smith, apontando algumas implicações que podem ser de interesse não apenas ao ensino baseado em modelização, mas também à aprendizagem de ciências de uma forma geral.

\section{Conceitualização, modelos no ensino de ciências e desenvolvimento cognitivo}

Para Matthews (2007), o que impeliu a pesquisa sobre modelos e modelização na área de ensino de ciências foram justamente os trabalhos de psicólogos e cientistas da cognição que reconheceram, na construção mental de modelos sobre o mundo, um papel central para a apropriação de conhecimento. Dentre os primeiros pesquisadores desse campo que enfatizaram a centralidade dos modelos, Matthews (2007) aponta a contribuição de Johnson-Laird, para quem 
A proposta da Redescrição Representacional como referencial ...

É agora plausível supor que modelos mentais têm um papel central e unificador em representar objetos, estados de sistemas, sequências de eventos, o modo como o mundo é, e as ações sociais e psicológicas da vida diária. Eles possibilitam a indivíduos fazer inferências e previsões, compreender fenômenos, decidir qual ação tomar e controlar sua execução, e, sobretudo experimentar eventos por representações; eles permitem usar a linguagem para criar representações comparáveis àquelas derivada do contato direto com o mundo; e eles relacionam palavras ao mundo por meio da concepção e percepção (JOHNSON-LAIRD, 1983, p. 397 apud MATTHEWS, 2007, p. 649, tradução nossa).

Adotando um conceito de modelo como uma representação análoga do mundo, Johnson-Laird considera-os como entidades teóricas que emergem da tentativa de dar sentido a inferências implícitas ou explícitas. A visão de modelos desse autor tem fundamentado numerosos trabalhos sobre modelos mentais no contexto do ensino, principalmente a partir dos anos 1990 (BORGES, 1998, 1999; BUCKLEY; BOULTER, 2000; MOREIRA, 1996; VOSNIADOU; BREWER, 1992). Inspirados nesse referencial, Greca e Moreira (2000) sintetizam sua relevância afirmando que compreender um fenômeno físico significa precisamente dispor de um modelo mental capaz de representá-lo.

O trabalho de Nersessian $(1992,1995)$ também constitui uma mirada aos modelos do ponto de vista cognitivo, em particular da cognição científica. Por essa especificidade, as contribuições da autora integram reflexões de natureza histórica, filosófica e epistemológica, como demonstra seu método histórico-cognitivo (NERSESSIAN, 1995). Essa autora argumenta que o raciocínio baseado em modelos (model-based reasoning) pode gerar mudança conceitual na ciência. A hipótese que ela propõe para explicar essa tese é a de que

[...] analogias, modelos visuais e experimentos de pensamento são prevalentes em períodos de mudança conceitual radical porque tal raciocínio baseado em modelos é um meio altamente eficaz de evidenciar e abstrair restrições de sistemas representacionais existentes e, à luz das restrições fornecidas pelo problema alvo, um meio eficaz de integrar restrições de múltiplas representações de tal forma que resultem novas estruturas representacionais (NERSESSIAN, 2002, p. 145, tradução nossa).

Esse processo envolveria diversas formas de abstração que, em conjunto, permitiram a geração, modificação, substituição e integração das restrições (constraints). Essas restrições poderiam ser fornecidas por diversos meios: verbais, matemáticos, imagísticos, pictóricos, físicos, cinestésicos, entre outros. Para essa autora, tanto a formação de conceitos quanto a mudança conceitual envolvem mudanças nas restrições (NERSESSIAN, 2002).

Ao abordarem o tema modelos da perspectiva da psicologia cognitiva e do desenvolvimento, Franco et al. (1997) apresentam e discutem as contribuições de três referenciais da área - a teoria dos modelos mentais de Johnson-Laird, a perspectiva de Nersessian (1992) e a teoria piagetiana - e traçam uma análise comparativa, visando articular as potencialidades das três perspectivas em direção a um modelo dos modelos mentais (FRANCO et al., 1997). 
Os autores ressaltam a oposição entre a abordagem piagetiana, que caracteriza a formação das imagens mentais na criança como subordinada à sua competência lógico-matemática, e a abordagem de Johnson-Laird (1990), que não aceita essa subordinação, mas coloca as imagens mentais como "correlatos perceptivos" dos modelos. Alinhando-se com essa última perspectiva no tocante a esse ponto, Franco et al. (1997) observam, contudo, uma lacuna na proposta de Johson-Laird, que é a de não ter desenvolvido satisfatoriamente uma teorização do processo de formação dos modelos mentais.

Um passo nessa direção seria dado por Nersessian (1992), ao voltar-se para sua análise histórico-cognitiva na busca por compreender como se daria a construção dos modelos científicos em termos de mecanismos e operações de pensamento. Com base nessa metodologia, a autora já indicava que a modelização envolvia técnicas de abstração, nomeadamente o raciocínio analógico, imagístico, experimentos de pensamento e análise de caso-limite, operações que são por ela examinadas tanto de um ponto de vista cognitivo quanto histórico. Nersessian (1995) diz que a modelização construtiva não é completamente formalizável como procedimento, mas as técnicas que dela fazem parte podem ser explicitadas, aplicadas e avaliadas. Dessa forma, Nersessian contribuía para preencher a lacuna da teoria dos modelos mentais apontada por Franco et al. (1997). A autora também indica como as chamadas técnicas de abstração relacionam-se com a aprendizagem em Física (NERSESSIAN, 1992).

O olhar de Nersessian $(1992,1995,2002)$ conduz-nos a pensar a produção, aplicação, adaptação e modificação de modelos sobre a realidade que nos cerca como uma atividade comum, mutatis mutandis, entre o trabalho do cientista e o desenvolvimento cognitivo dos sujeitos. $\mathrm{Na}$ busca por contribuições ao campo da didática das ciências naturais, parece-nos importante localizar confluências entre as discussões epistemológicas e as contribuições da psicologia do desenvolvimento ao problema da modelização.

Ao formular as questões postas na Introdução, pressupomos que existe, quer na evolução do pensamento científico quer no desenvolvimento cognitivo, uma passagem de estados de menor conhecimento para estados de maior conhecimento. Como ocorre essa passagem é justamente a questão central que motiva a epistemologia genética (CASTORINA; CARRETERO, 2014). Essa perspectiva central da obra piagetiana consiste em uma epistemologia que

[...] é naturalista sem ser positivista, que coloca em evidência a atividade do sujeito sem ser idealista, que se apóia igualmente no objeto ao mesmo tempo que o considera um limite (portanto, existindo independentemente de nós mas sem ser completamente alcançado) e que, sobretudo, vê no conhecimento uma construção contínua [...] (PIAGET, 2012, p. 6).

Uma das questões que divide as diversas teorias do desenvolvimento refere-se ao alcance dos processos que são postulados para explicar essa passagem: seriam eles de domínio geral ou de domínio específico? Trata-se de outro aspecto que distancia as abordagens de Piaget, orientado na primeira opção, e de Nersessian (1992), mais inclinada para a segunda.

Do ponto de vista do desenvolvimento, um domínio é o conjunto de representações que sustenta uma área específica de conhecimento, tal como a Linguagem, a Matemática ou a Física. Dentro de cada domínio é possível distinguir microdomínios, subconjuntos de um certo 
domínio. Pode-se assim pensar na gravitação como um microdomínio da Física, ou na geometria como um microdomínio na Matemática. No modelo piagetiano de desenvolvimento, grandes mudanças acontecem de forma aproximadamente simultânea em diferentes domínios: mudanças de estágios de desenvolvimento implicariam em alterações nas estruturas lógicas de pensamento dos sujeitos, e estas se aplicariam tanto ao pensamento físico quanto ao matemático, biológico, moral, e assim por diante. Assim é que o modelo piagetiano concebe os mecanismos de mudança entre os estágios como sendo de domínio geral. Tal abordagem confere aos processos cognitivos na visão piagetiana uma independência em relação ao conteúdo e ao contexto, ponto este criticado por Franco et al. (1997).

Outros autores, no entanto, defendem a especificidade de domínio dos processos cognitivos. Nessa visão, as mudanças cognitivas ocorrem dentro de um domínio, não necessariamente ao mesmo tempo que em outros domínios. Enquanto determinado tipo de transição ocorre, por exemplo, no que diz respeito à aquisição da linguagem, a mesma transição poderá ocorrer no domínio da física apenas em momentos posteriores do desenvolvimento. É fácil notar que, justamente pela sua utilização de uma análise histórico-cognitiva cuidadosa, com atenção aos problemas específicos enfrentados pelos cientistas e à natureza dos próprios conteúdos de conhecimentos científicos, a contribuição de Nersessian orienta-se num sentido mais voltado à especificidade dos processos cognitivos, respondendo assim à segunda questão problemática da teoria piagetiana posta por Franco et al. (1997).

A especificidade de domínio é, por vezes, associada a perspectivas inatistas. Nessa linha, é muito influente o trabalho de Jerry Fodor, $A$ modularidade da mente (FODOR, 1983). Para esse autor, a mente seria formada por "módulos", ou sistemas de entrada inatos que funcionam de forma independente e seriam encapsulados, isto é, teriam seu funcionamento indisponível ao restante do aparelho cognitivo. Entre outros nomes, as psicólogas Elizabeth Spelke e Susan Carey também produziram importantes contribuições à pesquisa em desenvolvimento na linha da especificidade de domínio, dentro de uma perspectiva inatista. No entanto, admitir a existência de processos específicos de domínio não implica uma adesão completa ao inatismo, ou mesmo a convicção de que nada há de domínio geral no desenvolvimento.

Por exemplo, argumentando contra a concepção de desenvolvimento de Fodor, Karmiloff-Smith (1992) rejeita a tese inatista de módulos pré-especificados com os quais a mente já estaria equipada desde o início. No lugar disso, Karmiloff-Smith propõe que o desenvolvimento envolve uma progressiva modularização da mente, que ocorreria de forma gradual. Essa autora defende que o desenvolvimento deve ser compreendido como uma relação intricada entre processos de domínio geral (mais do que é aceito pelos inatistas) e outros de domínio específico (mais do que é aceito pelos piagetianos). Na próxima seção apresentaremos a proposta da Redescrição Representacional e, a seguir, apontamos algumas replicações e variações de seus estudos, além de algumas críticas a ela dirigidas.

Ao discutir algumas implicações da proposta de Karmiloff-Smith ao contexto da construção de modelos no ensino de ciências, destacaremos principalmente três aspectos: a consideração do caráter implícito de certos conhecimentos; o papel relevante que a autora atribui à sobregeneralização, entendida como a desconsideração das propriedades dos objetos reais para a elaboração das representações; e a flexibilidade cognitiva, isto é, a crescente disponibilização dos conhecimentos que o sujeito produz em situação a outras partes do seu aparelho cognitivo. Essa última categoria poderia ajudar a compreender de que modo os conhecimentos 
produzidos em determinada situação são transpostos para outras situações e, no caso dos modelos, como eles poderiam passar a ser reconhecidos como integrando uma estrutura conceitual mais ampla.

\section{A proposta da Redescrição Representacional}

Buscando compreender inter-relações entre sequências de ações e teorias implícitas em processos de desenvolvimento cognitivo, Karmiloff-Smith e Inhelder (1975) propuseram a crianças entre 4 a 9 anos a tarefa de equilibrar blocos com diferentes distribuições de massa sobre uma barra estreita. Alguns blocos eram simétricos e homogêneos, outros eram visivelmente assimétricos (tinham um pequeno peso colado em uma de suas extremidades) e um terceiro grupo consistia em blocos aparentemente simétricos, visualmente idênticos aos primeiros, mas que tinham sido preenchidos com um pouco de chumbo em uma de suas extremidades. As autoras observam que as crianças nessa faixa etária não apenas constroem, mas também generalizam teorias para a condição de equilíbrio, e que o fazem sem procurar espontaneamente por contraexemplos, inclusive frequentemente ignorando-os quando são encontrados. Apenas gradualmente é que passam a reconhecer os contraexemplos que se apresentam. Dentre os aspectos apontados pelas autoras nesse estudo, destacamos dois que se relacionam diretamente com os modelos: a produção de teorias e a sobregeneralização.

Construir - e, principalmente, generalizar - teorias-em-ação mostra-se, na análise daquelas autoras, como uma tendência que se apresenta subjacente à atividade dos sujeitos naquela situação, que pareciam buscar produzir explicações simples, gerais e unificadas, isto é, que fossem potencialmente aplicáveis a todos os elementos de seu domínio. Em certo momento durante o desenvolvimento, a teoria-em-ação segundo a qual o ponto de equilíbrio coincide com o centro geométrico do bloco mostra-se profundamente imbuída nos esquemas de ação dos sujeitos. Karmiloff-Smith e Inhelder (1975) relatam então que, após tentativas bem-sucedidas de equilibrar o bloco sobre seu centro geométrico, ao experimentar o insucesso dessa teoria com outros blocos, os sujeitos tendem a apegar-se à teoria inicial tanto quanto possível, muitas vezes tentando equilibrar o bloco sobre seu centro geométrico de forma mais cuidadosa ou, eventualmente, recusando a tarefa por considerar que aqueles blocos são impossíveis de equilibrar.

Assim como a construção de teorias falsas, a sobregeneralização não significa um impedimento ao desenvolvimento, mas, pelo contrário, representa um processo criativo. Os autores destacam que

\footnotetext{
Sobregeneralização, um termo às vezes depreciativo, pode ser visto como uma simplificação criativa de um problema ao ignorar alguns dos fatores complicadores [...] Sobregeneralização não é apenas um meio de simplificar, mas também de unificar; não é, portanto, surpreendente que as crianças e os cientistas frequentemente recusem contra-exemplos, já que eles complicam o processo unificador (KARMILOFF-SMITH; INHELDER, 1975, p. 209, tradução nossa, grifo dos autores).
}

Por exemplo, no problema do equilíbrio dos blocos, o peso é uma variável inicialmente desconsiderada pelos sujeitos, que focam sua atenção no comprimento dos blocos. E, de fato, 
para muitos dos blocos - assim como para situações de equilíbrio de outros objetos na vida cotidiana - a consideração do tamanho pode bastar para obter sucesso com a tentativa de equilíbrio. Aí se encontra a relevância da sobregeneralização no processo de desenvolvimento, pois a surpresa experimentada pelo sujeito frente ao bloco que não se equilibra apoiado pelo centro geométrico parece ocorrer apenas quando aquela previsão deriva de uma teorização já consolidada. Nesse sentido, a sobregeneralização parece desempenhar uma função criativa para o questionamento das teorias-em-ação do sujeito e eventual desenvolvimento de novas teorias-em-ação. Esse processo é implícito no caso das crianças, mas poderia ser intencional no caso dos cientistas (KARMILOFF-SMITH; INHELDER, 1975). Esse exemplo também permite ilustrar a maneira pela qual a abstração toma parte na produção de teorias-em-ação dos sujeitos: ao desconsiderar uma das variáveis do problema que se torna possível a sobregeneralização necessária à formação e à unificação da teoria-em-ação mobilizada nessa situação.

Em um trabalho posterior, Karmiloff-Smith propõe uma concepção para o desenvolvimento cognitivo fundamentada na hipótese de que a construção do conhecimento se dá pela possibilidade de a mente explorar, internamente, as informações com que está equipada (quer seja pela via inata, quer adquirida na interação com o ambiente externo), ao redescrever suas representações, isto é, ao reelaborar sucessivas representações internas, em diferentes formatos (ou níveis). Através desse processo, a informação implícita na mente transformarse-ia progressivamente em conhecimento explícito para a mente. Tal processo é chamado pela autora de Redescrição Representational (RR) (KARMILOFF-SMITH, 1992).

A existência de um processo de RR, por si mesma, significa apenas que as representações internas passariam por uma recodificação, em diferentes formatos, ao longo do desenvolvimento. Adicionalmente, Karmiloff-Smith (1992) propõe também um modelo para os formatos ou níveis, além de analisar a dinâmica de transição entre eles. Neste modelo, a autora propõe a existência de pelo menos quatro níveis, cada um deles representando um formato no qual o conhecimento é representado e re-representado: Implícito (I), Explícito 1 (E1), Explícito 2 (E2) e Explícito (E3). No nível I, as representações estariam codificadas em um formato procedimental, que possibilita ao sujeito usá-las para analisar e responder a estímulos externos. Essas representações são fragmentadas em relação ao universo de conhecimento do sujeito: não formam ligações com o restante do domínio ao qual pertencem. Com isso, permanecem indisponíveis para outros operadores no sistema cognitivo do sujeito.

A redescrição dessas representações procedimentais, que culminaria no formato representacional E1, é descrita pela autora como um processo de abstração "[...] em linguagem de alto nível” (KARMILOFF-SMITH, 1992, p. 21, tradução nossa). Seriam descrições reduzidas, que abandonam vários detalhes das informações perceptuais. Assim, por exemplo, uma placa de trânsito mostra uma imagem icônica para avisar que há zebras na estrada. Claramente, o estímulo de entrada recebido pelo sistema perceptivo do sujeito frente a uma zebra real é bastante diferente daquela imagem; ainda assim, o sistema cognitivo permite relacioná-la a uma zebra real. A partir do nível E1, as representações são conceituais. Em comparação às representações do nível I, estas são mais simples e cognitivamente flexíveis. Pelo fato de serem conceituais, decorre que não sejam mais tão fragmentadas; estão acessíveis ao restante do sistema cognitivo para serem manipuladas e relacionadas com outras representações. Daí decorre que é a partir do nível E1 que se inicia o processo de construção de teorias-em-ação. No entanto, isso não significa que estejam acessíveis à consciência, e ainda menos que sejam passíveis de verbalização. 
É no nível seguinte da RR que essas representações tornam-se acessíveis à consciência, constituindo assim o formato correspondente ao nível E2. Mas o modelo proposto por Karmiloff-Smith pressupõe que consciência não possa ser reduzida à verbalização. É esse aspecto que diferencia os níveis E2 e E3: embora as representações em ambos os níveis sejam conscientes, é apenas no último que elas se tornam disponíveis ao relato verbal. É assim que, por vezes, desenhamos diagramas de coisas que não conseguimos verbalizar; ou, em certos momentos do desenvolvimento, o sujeito experimenta uma sensação do tipo eu sei, mas não sei explicar. No caso da conceitualização no domínio da Física, notamos que não é infrequente que um estudante seja capaz de resolver corretamente um problema, mas encontre grandes dificuldades ao produzir uma explicação, oral ou escrita, sobre como o fez.

Já as representações no formato do nível E3 são próximas o suficiente da linguagem natural para que possam ser traduzidas de forma comunicável. Há dois aspectos desse formato que devem ser destacados. Em primeiro lugar, Karmiloff-Smith (1992) mantém aberta a possibilidade de que certos conhecimentos, obtidos pela interação entre os sujeitos através da via linguística, sejam diretamente codificados nesse formato, sem terem antes passado pelos níveis anteriores. Por outro lado, a autora aponta também a possibilidade de que alguns conhecimentos obtidos dessa forma não consigam se ligar ao restante das representações, armazenadas no aparelho cognitivo em outros formatos. Essa última observação também nos parece interpretável no contexto da conceitualização em Física, já que a simples enunciação verbal de leis ou princípios claramente não conduz, necessariamente, ao sucesso do sujeito na interpretação de uma situação ou na resolução de um problema.

Mas como acontecem as transições de um nível para outro? Karmiloff-Smith (1992) defende que esse processo envolve três fases recorrentes. Na primeira fase, o foco do sujeito é nas informações do ambiente externo e sua ação é orientada pelos dados. A partir desses dados, ele cria adjunções representacionais, isto é, representações armazenadas independentemente, que podem ser acrescentadas ao rol disponível ao sujeito, mas não estão ligadas a outras representações anteriores e já estáveis. O ponto culminante da fase 1 é o sucesso consistente do sujeito em desempenhar com êxito uma tarefa, seja equilibrar blocos, amarrar os cadarços, produzir uma forma linguística corretamente, ou qualquer outra do microdomínio considerado. É o que Karmiloff-Smith (1992) refere-se como behavioral mastery, e ele é sustentado pelas adjuções representacionais.

Mas esse sucesso da conduta do sujeito na realização de uma tarefa não constitui um fechamento do desenvolvimento cognitivo naquele microdomínio. Segue-se a ele uma segunda fase, durante a qual o foco do sujeito deixa de ser nos dados externos e passa para as mudanças nas representações internas. É durante essa fase que passaria a ocorrer uma desconsideração das propriedades do ambiente externo, tais como o ato de ignorar parte das propriedades e fatores complicadores que existem naquele ambiente. Segundo Karmiloff-Smith (1992), essa desconsideração pode - mas não necessariamente irá - reduzir o sucesso do sujeito na realização da tarefa. Eventualmente, um equilibrio é alcançado entre os controles externo e interno. Esse ponto é colocado pela autora como uma terceira fase do desenvolvimento. Nela, as representações internas são conciliadas com os dados recebidos do ambiente externo. O resultado pode conduzir a uma conduta semelhante à observada durante a fase 1 , isto é, ao sucesso na realização da atividade proposta.

Um aspecto interessante dessa dinâmica é o papel atribuído ao conflito cognitivo. $\mathrm{O}$ modelo de RR proposto por Karmiloff-Smith (1992) reconhece um papel importante exercido 
pelo feedback negativo (lacunas e contradições) dentro de uma fase, pois através desse feedback o sujeito produziria as adjunções representacionais que culminariam com a behavioral mastery. Contudo, na passagem de uma fase para outra, é o feedback positivo que tem um papel crucial: apenas as representações que tenham atingido um estado estável é que sofrem uma redescrição representacional. Esse é outro ponto de afastamento entre a proposta de Karmiloff-Smith e a visão piagetiana, para a qual um sistema em equilíbrio não iria progredir espontaneamente.

A RR poderia ser entendida assim como “[...] um processo de 'apropriação' de estados estáveis para extrair as informações que eles contêm, que podem então ser usadas de forma mais flexível para outros propósitos" (KARMILOFF-SMITH, 1992, p. 25, tradução nossa). Esse processo reiterativo, ao invés da simples interação com o ambiente externo, é que seria responsável pelo aumento da flexibilidade cognitiva e pela eventual emergência das representações ao nível consciente.

\section{Replicações e críticas à proposta da $R R$}

Desde a proposição do processo e do modelo de RR, diversos grupos de pesquisa em desenvolvimento têm replicado o experimento do equilíbrio dos blocos utilizado por Karmiloff-Smith (1992). Karen Pine e David Messer desenvolveram sistematicamente diversas variações e extensões do desenho original pelo menos desde 1998 (MESSER; PINE; BUTLER, 2008; PINE; MESSER, 1998, 1999, 2003). Em um desses estudos, um grupo de 25 crianças era apresentado a um conjunto de tarefas de equilíbrio de blocos sobre um fulcro a cada dia, por cinco dias consecutivos. Os níveis das representações, originalmente em número de quatro (KARMILOFF-SMITH, 1992), foram transformados em sete: implícito; transição do implícito, abstração não-verbal; abstração verbal; transição do explícito; explícito E3 e explícito E4. Esses níveis correspondem a uma descrição mais gradual e extensa, mas no mesmo direcionamento da proposta de Karmiloff-Smith. Os dois níves de abstração (verbal e nãoverbal) corresponderiam ao nível E1, e evidência sugerindo a existência deste foi encontrada no estudo. Para o nível E2, que consistiria na habilidade de equilibrar tanto blocos simétricos quanto assimétricos de forma consciente e sem verbalização, não foi encontrada nenhuma evidência. Por fim, o nível E3, que nesse estudo foi dividido em E3 e E4, também foi corroborado por meio dos dados. Os autores endossam a existência de um processo de re-elaboração das representações dos sujeitos iniciando-se em um nível implícito e progredindo para novos formatos, passando por alguma forma de abstração.

Em um trabalho posterior, Messer, Pine e Butler (2008) aplicaram um teste no qual duas tarefas eram propostas aos participantes: uma delas consistia em equilibrar pesos a diferentes distâncias sobre uma tábua horizontal equilibrada por um fulcro, como uma espécie de balança (SIEGLER, 1976) e a outra consistia numa versão do experimento de equilíbrio proposto por Karmiloff-Smith (1992), acrescido da tarefa de prever se determinadas configurações do sistema (em termos da distribuição dos blocos em torno do fulcro) iriam resultar em equilíbrio ou desequilíbrio. O objetivo desses pesquisadores era o de avaliar se o contexto específico da tarefa proposta ao sujeito interfere na sua performance. Assim, embora ambas as tarefas envolvam o equilíbrio de corpos, elas não são idênticas. Participaram deste experimento 86 crianças com idades entre 5 e 7 anos. Os níveis das representações das crianças foram assim classificadas: crianças que obtiveram sucesso em equilibrar pelo menos dois blocos simétricos 
e dois assimétricos, apresentaram explicações implícitas e não usaram as palavras peso e distância em mais de um problema foram categorizadas no nível Implícito (nível I); aquelas que equilibraram pelo menos dois blocos simétricos e no máximo um assimétrico, no nível Abstração; (semelhante ao nível E1) e aquelas que equilibraram pelo menos dois blocos simétricos e dois assimétricos, além de usar as palavras peso e distância para explicar ao menos dois problemas, foram classificadas no nível Explícito (semelhante ao nível E2/3). Os pesquisadores destacam que os participantes não se valeram dos mesmos processos cognitivos nas diferentes tarefas: as próprias características da tarefa parecem ter influenciado consideravelmente a performance das crianças (MESSER; PINE; BUTLER, 2008).

Os resultados desse último estudo também levaram os autores a questionar a caracterização do nível (I) conforme proposto por Karmiloff-Smith, pois, ao solicitar às crianças uma previsão do comportamento dos blocos, elas foram capazes de produzir explicações explícitas e de utilizar-se de regras nessas explicações. Esses resultados, entre outros, foram interpretados pelos pesquisadores como um indicativo de que o desenvolvimento não consiste em um processo linear, mas que existiria um conjunto multidimensional de processos cognitivos, cujo uso pelo sujeito é variável conforme as particularidades da tarefa proposta. Com base nessas considerações, Messer e seus colaboradores destacam a dificuldade em classificar crianças em termos de estágios ou níveis gerais, mesmo quando essa análise é restrita a um único domínio, e concluem sugerindo que uma compreensão melhor sobre o desenvolvimento poderia ser alcançada identificando-se dimensões do processamento de informação relevantes a um dado domínio (como por exemplo a codificação) e os contextos nos quais essas dimensões operam (MESSER; PINE; BUTLER, 2008).

Em sua tese de doutorado, Butler (2007) apresenta uma série de estudos em torno da perspectiva da RR de Karmiloff-Smith que, além de apontar parte das indicações publicadas em Messer, Pine e Butler (2008), corrobora algumas das observações de Karmiloff-Smith acerca dos mecanismos de mudança. Um dos pontos destacados é a existência de uma estabilidade no nível representacional das crianças participantes que antecede (embora não seja, necessariamente, imediatamente anterior) a mudança representacional. Conforme já foi discutido, esse é um dos aspectos de afastamento entre a proposta da RR com relação à visão piagetiana do desenvolvimento, que enfatizava a necessidade da instabilidade das representações como motor da mudança. O estudo também apresenta alguns avanços sobre a proposta original, em especial denotando um papel-chave da verbalização no processo de mudança cognitiva.

\section{Possíveis contribuições da $\mathbf{R R}$ à compreensão do processo de Conceitualização de modelos na Educação Científica}

Antes de discutir a possibilidade de derivar contribuições da proposta da RR para o contexto da educação científica, faz-se necessário considerar alguns aspectos da proposta em si. Primeiramente, deve-se notar que o processo de ensino-aprendizagem escolar não foi, diretamente, o objeto específico da teoria da RR no momento de sua formulação. A área de formação e de atuação profissional de Karmiloff-Smith é o campo da psicologia, em especial na linha dos distúrbios do desenvolvimento, com grande número de publicações sobre a Síndrome de Williams. Beyond modularity, a obra na qual apresenta a proposta da RR, concentra-se na questão sobre "[...] o que a perspectiva do desenvolvimento pode oferecer à ciência 
cognitiva" (KARMILOFF-SMITH, 1992, p. xiii, tradução nossa). Não se trata, portanto, de uma obra voltada primariamente à didática. Por outro lado, a RR é também uma hipótese sobre como a mente humana explora e organiza o conhecimento de tal modo a possibilitar um auto -enriquecimento. É nesse sentido que nos parece muito pertinente à tentativa de compreender a conceitualização de modelos científicos escolares, já que a construção destes envolve, entre outros fatores, uma atividade complexa do aparelho cognitivo.

Um dos pontos de interesse comum entre a proposta da RR e a investigação sobre a conceitualização de modelos no contexto educacional refere-se à existência de conhecimentos implícitos, suas funções ao longo da apropriação conceitual e o seu processo de explicitação. Sabe-se, por exemplo, que as chamadas concepções alternativas possuem um caráter eminentemente implícito (NARDI; GATTI, 2008) e que também podem ser bastante efetivas nas situações cotidianas. Pensar que a força é algo que se extingue espontaneamente, ou que o corpo mais pesado cai mais depressa, ou ainda que materiais isolantes são capazes de nos aquecer, pode conduzir ao sucesso no enfrentamento de pequenas tarefas como abrigar-se do frio ou jogar uma bola para alguém em um jogo. Essas representações tem, assim, o potencial de produzir behavioral mastery. De fato, o próprio exemplo da teoria de equilibração dos corpos pelo centro geométrico, estudada por Karmiloff-Smith (1992), consiste, também, em um bom exemplo de concepção alternativa.

Na pesquisa em ensino de ciências, surgiram nas décadas de 1980 e 1990 muitos estudos visando a superação das concepções alternativas com base na explicitação destas, seguida de um momento de "conflito cognitivo", que introduziria um feedback negativo às representações e assim, acreditava-se, abriria caminho para a "mudança conceitual” (POSNER et al., 1982). No entanto, após o desenvolvimento de um extenso programa de pesquisa na área, essa estratégia não pareceu funcionar, ou, no mínimo, não tão bem quanto esperado. Uma série de motivos, de diferentes ordens, pode ser apontada como possíveis explicações para a ineficácia dessa proposta (LIMÓN, 2001). Mesmo quando ocorre uma aparente mudança conceitual, não há garantias de que isso signifique ter havido uma troca de concepções, extirpando a antiga e colocando uma nova em seu lugar: é possível que esteja ocorrendo apenas uma seleção de concepções de acordo com o contexto (DUIT; TREAGUST, 2003; MORTIMER, 1996).

Embora também pressupondo uma explicitação progressiva, o modelo de RR de Karmiloff-Smith (1992) não vai ao encontro das propostas de mudança conceitual. Na visão clássica da mudança conceitual, uma representação que possa ser problematizada deve ser explícita ou, no mínimo, consciente. Assim, Posner et al. (1982) falam de uma conscientização (awareness) do estudante em relação às suas assunções fundamentais que precederia a mudança conceitual. Em contraste, Karmiloff-Smith (1992) defende a ocorrência de uma redescrição que incide diretamente sobre representações no nível implícito, o que já implica em um processo de transformação dessas representações de um formato para outro. Mais do que isso, ela postula a existência de um nível que não é implícito, nem totalmente explícito, nem disponível ao acesso consciente, e que corresponde ao formato E1. Uma implicação importante dessa hipótese é a de que o status das representações do sujeito não poderia ser acessado apenas com base na interação linguística. E a diferenciação entre as representações I e E1 seria relevante, pois apenas no último caso elas estariam disponíveis para o restante do conteúdo do aparelho cognitivo. Além disso, Karmiloff-Smith aponta que a acumulação de anomalias não leva necessariamente à elaboração de uma nova representação, ao passo que a emergência de uma nova 
representação também não requer, necessariamente, o recebimento de novos dados (anômalos ou não) do ambiente externo (KARMILOFF-SMITH, 1992).

Especificamente no contexto do ensino de ciências, tomando-se o aspecto reiterativo do processo de RR e a observação de Messer, Pine e Butler (2008) dos diferentes processos cognitivos e diferentes representações desenvolvidas pelas crianças de forma variável de acordo com a tarefa proposta, também é possível vislumbrar um panorama um pouco mais amplo para a questão da convivência de diferentes representações. Após o abandono da expectativa de produzir-se uma mudança conceitual súbita pela via do conflito cognitivo, algumas pesquisas da área passaram a considerar que as concepções espontâneas ou alternativas continuam coexistindo com as concepções científicas, introduzidas pela educação escolar (e.g., Shtulman e Valcarcel, 2012). Notamos que essas pesquisas tendem a considerar como polos opostos as representações espontâneas e as científicas, sendo a questão de fundo descobrir como trazer o estudante para mais próximo das últimas.

Esse direcionamento parece trazer implicitamente a noção de que as representações científicas são unívocas, isto é, cada conceito físico teria um único significado, ao qual cabe ao estudante apropriar-se, se não substituindo sua concepção alternativa, pelo menos acrescentado a científica ao seu rol e utilizando-a quando o contexto assim o exige. Mas esse não parece ser o caso dos conceitos científicos. Assim como as diferentes situações suscitam nas crianças a produção de diferentes representações, parece-nos que os conceitos científicos também podem se revestir de significados diversos. Isso ocorre não apenas ao longo da história de um conceito - por exemplo, no caso dos conceitos historicamente aceitos de força (COELHO, 2010) - mas também dentro de um mesmo período histórico - tal como no caso dos significados do conceito de massa na física contemporânea (JAMMER, 2000).

Isso significa dizer que não são apenas as representações espontâneas - geralmente vistas como o alvo da mudança conceitual - que são múltiplas, mas também as científicas tidas em geral como o produto desejável dessa mudança. Tal multiplicidade é um dos aspectos que parece inspirar, por exemplo, a proposta do perfil conceitual (MORTIMER, 1996). Essa observação, em conjunto com os resultados empíricos do estudo de Messer, Pine e Butler (2008) aponta que não se trata apenas de uma distinção entre contextos nos quais o conhecimento científico é ou não é necessário, mas de uma consideração do papel das diferentes situações - no sentido atribuído por Vergnaud (2013) - na mobilização de diferentes modelos científicos (ou não-científicos) para um mesmo fenômeno. Para Vergnaud, situações são tarefas a resolver e é a elas que os esquemas de pensamento dos sujeitos adaptam-se no processo de desenvolvimento, de modo que diferentes classes de situações mobilizam diferentes conceitos para certo objeto, fenômeno ou operação. Por exemplo, a adição pode ser compreendida como uma contagem do total; como um crescimento a partir de um valor inicial; ou como uma comparação entre medidas, entre outras possibilidades, de acordo com a situação enfrentada pelo sujeito (VERGNAUD, 1986).

Adicionalmente, a discussão sobre o implícito e o explícito tem um interesse para a pesquisa em ensino que se estende para muito além da mudança conceitual. Dificilmente se poderia negar que o conhecimento individual dos sujeitos tem uma parcela considerável de conteúdo implícito, tanto nos aspectos relacionados aos objetos da ciência (POZO; GÓMEZ CRESPO, 2005) quanto naqueles relacionados à natureza da ciência (CLOUGH, 2006). Augé (2014) destaca imbricações entre as representações implícitas e outros aspectos da cognição, 
em especial as restrições cognitivas e a especificidade de domínio. À luz da proposta da RR, nem o implícito, nem o explícito, exclusivamente, poderiam ser colocados como objetivos finais do processo de conceitualização. Não apenas porque trata-se de um processo reiterativo de reelaboração das representações, mas também porque, no contexto do ensino-aprendizagem, é desejável tanto a capacidade de comunicação dos conhecimentos (representada pelo nível E3), como também a sua acessibilidade ao corpo de conhecimentos do sujeito como um todo e à sua capacidade de operar com essa representação em situações (tarefas) que a envolvam, o que não é necessariamente garantido pelo formato representacional E3.

Tal acessibilidade está ligada à propriedade de uma representação de apresentar algum grau de flexibilidade cognitiva. Notamos que essa flexibilidade é importante para o contexto do ensino-aprendizagem de ciências, pois

[...] o modelo RR postula que representações através de diferentes códigos só podem ser ligadas se as representações estiverem todas definidas explicitamente, pelo menos, em um formato E1. Portanto, para o conhecimento de física ser relacionado com o conhecimento matemático, o último deve também sofrer redescrição representacional (KARMILOFFSMITH, 1992, p. 89, tradução nossa).

Essa observação pode auxiliar, pelo menos em parte, a interpretar a dificuldade encontrada pelos estudantes em apropriarem-se da Matemática como uma estruturante do conhecimento físico (PIETROCOLA, 2002). Isso ocorreria porque não se trata de uma mera aplicação de conhecimentos matemáticos a um contexto "aplicado" na Física, como foi notado pelo autor citado, mas sim da necessidade de redescrever um conjunto de representações, atribuindo-lhes novos significados. Pode assim ocorrer que, estando em um nível implícito, tais representações até contribuam para algum sucesso dos sujeitos no enfrentamento de situações dentro do domínio da Matemática (behavioral mastery). Contudo, o obstáculo que se interpõe aos estudantes na busca por uma apropriação do caráter estruturante desses conhecimentos matemáticos no contexto da física (KARAM; PIETROCOLA, 2009) parece ser, de um ponto de vista cognitivo, a necessidade de tornar esses conhecimentos disponíveis a outros domínios, isto é, torná-los mais cognitivamente flexíveis. Dado que esse processo implica em uma redescrição representacional, não deveria causar qualquer surpresa o fato de ele não ocorrer automaticamente, mas frequentemente impor dificuldades consideráveis aos estudantes.

No âmbito das questões relacionadas à modelização, a proposta da RR estabelece algumas relações interessantes. Consideremos, inicialmente, a função atribuída à abstração nesse processo. No caso da passagem das representações do nível implícito para o nível explícito E1, Karmiloff-Smith (1992, p. 21) propõe que essa redescrição é, ela própria, uma abstração. Embora não haja na obra uma definição exata do que a autora compreende por "abstração", aparentemente o significado é bastante próximo daquele que tipicamente é atribuído nas discussões sobre modelos e modelização no contexto do ensino de Ciências. Algumas das expressões empregadas pela autora para descrever o nível mais abstrato são "descrições reduzidas, que perdem vários detalhes da informação codificada procedimentalmente"; "mais simples"; é também uma representação que "perdeu muitos detalhes perceptuais" (KARMILOFFSMITH, 1992, p. 21, tradução nossa). Certamente, no contexto da apropriação individual do 
conhecimento esse processo não é intencional, pelo menos não no caso de crianças pequenas. Não está totalmente claro, na proposta de Karmiloff-Smith, se a produção de abstrações é uma tendência para a qual o desenvolvimento conceitual se dirige, ou se é um subproduto deste. Ainda assim, fica claro que ela toma parte no processo de redescrição de uma representação implícita para um formato mais flexível.

Outro ponto convergente com as discussões sobre abstração no contexto da modelização é a observação de que esse processo resulta em representações que são produtivas, no sentido de já não serem mais encapsuladas, mas sim manipuláveis e disponíveis para o conjunto das demais representações E1, ou seja, transportáveis para outros contextos. Assim, enquanto no caso da produção de conhecimento científico a abstração se faz necessária para elaborar contrapartes conceituais dos objetos reais, de modo que esses conceitos possam ser articulados com modelos e teorias da ciência, no caso da conceitualização ela parece exercer um papel semelhante, ao possibilitar a emergência de representações passíveis de serem ligadas ao restante do universo de representações do sujeito, tanto intra quanto interdomínio. Sobre o segundo caso, Karmiloff-Smith (1992) postula ainda que a RR pode ocorrer espontaneamente, sem que nenhuma análise de novos dados esteja ocorrendo, e até mesmo sem nenhum tipo de pressão externa.

Essa flexibilidade cognitiva pode, ainda, ser investigada nas representações dos sujeitos comparando-se diferentes modelos teóricos (BUNGE, 1977) específicos de determinados fenômenos (por exemplo, o modelo do pêndulo simples, ou do sistema massa-mola) relacionados a uma mesma teoria geral (por exemplo, a Mecânica Newtoniana). Seriam os princípios que sustentam determinada teoria geral, reconhecidos como pertinentes pelos sujeitos, em situações relacionadas com diferentes modelos teóricos? Ou estariam esses princípios codificados em formatos encapsulados, sendo mobilizados apenas para um pequeno conjunto de modelos? Essa indagação é relevante, pois incide justamente sobre o caráter de um princípio de funcionar como um guia heurístico (CUSTÓDIO; PIETROCOLA, 2004), permitindo a mobilização dos saberes para outros contextos. Além disso, os princípios ou teorias gerais também são responsáveis por favorecer uma unificação dos conhecimentos, formando um sistema de pensamento coerente.

O mesmo tipo de questionamento poderia ser feito relativamente ao status de determinados objetos-modelo (BUNGE, 1977), isto é, das representações conceituais de objetos reais, criadas a partir de idealizações e abstrações operadas pelo sujeito epistêmico sobre esses objetos. A noção de centro de massa, por exemplo, seria mobilizada pelos sujeitos para explicar o equilíbrio de corpos inomogêneos tanto quanto nas explicações relacionadas ao movimento de projéteis? Ou receberia ela diferentes sentidos de acordo com as situações propostas? Em que medida os estudantes conseguem flexibilizar os objetos-modelo estudados para novas situações? A priori, não parece haver razões para esperar que as respostas sejam as mesmas para os diferentes microdomínios das ciências empíricas. Isto é, mesmo admitindo-se a existência de um processo geral de RR, os modelos específicos de organização dos níveis podem ser distintos em cada microdomínio. Ainda assim, se a flexibilidade cognitiva for considerada desejável como parte da educação científica, parece-nos que as questões levantadas aqui podem contribuir para pensar-se em estratégias didáticas que a favoreçam. 


\section{Considerações Finais}

Embora se trate de um referencial praticamente ausente na literatura de pesquisa em educação científica, nesse trabalho pudemos mostrar algumas potencialidades da proposta da RR de Karmiloff-Smith para essa área de investigação. Por termos um olhar mais preocupado com questões relativas à modelização no ensino, nosso estudo da obra da autora conduziunos a destacar os aspectos do implícito, da sobregeneralização e da flexibilidade cognitiva, e foi possível apontar imbricações desses aspectos em diversas questões importantes envolvidas naquela temática.

Em uma perspectiva mais geral, à luz da proposta de Karmiloff-Smith, avanços na área de ensino de ciências - em particular na linha de aprendizagem científica - poderiam partir de investigações que buscassem caracterizar processos de RR ao longo da conceitualização de conteúdos ou temas específicos dos campos das ciências naturais. Ao mesmo tempo, a proposta também levanta questões investigativas pertinentes à educação científica e que transcendem as especificidades de conteúdo, como aquelas que formulamos na seção precedente. Dado que se trata de um modelo de níveis, e não de estágios, a proposta de Karmiloff-Smith não se limita ao desenvolvimento infantil, podendo ser aplicada a qualquer idade, incluindo adultos.

\section{Referências}

AUGÉ, P. S. Restrições cognitivas e o desenvolvimento na história da ciência e no indivíduo das concepções sobre queda dos corpos e ação física. 2014. Tese (Doutorado em Educação) - Universidade Federal Fluminense, Niterói, 2014.

BORGES, A. T. Como evoluem os modelos mentais. Ensaio, Belo Horizonte, v. 1, n. 1, p. 66-92, 1999.

BORGES, A. T. Modelos mentais de eletromagnetismo. Caderno Brasileiro de Ensino de Física, Florianópolis, v. 15, n. 1, p. 7-31, 1998.

BUCKLEY, B. C.; BOULTER, C. J. Investigating the role of representations and expressed models in building mental models. In: GILBERT, J. K.; BOULTER, C. (ed.). Developing models in science education. Dordrecht: Springer, 2000. p. 119-135.

BUNGE, M. Treatise on basic philosophy: ontology I: the furniture of the world. Dordrecht: Reidel, 1977.

BUTLER, C. Evaluating the utility and validity of the representational redescription model as a general model for cognitive development. 2007. Thesis (Doctor in Philosophy) - University of Hertfordshire, Hatfield, 2007.

CASTORINA, J. A.; CARRETERO, M. Desenvolvimento cognitivo e educação: o início do conhecimento: volume 1. Porto Alegre: Penso Editora, 2014. 
CLOUGH, M. P. Learners' responses to the demands of conceptual change: considerations for effective nature of science instruction. Science \& Education, Dordrecht, v. 15, n. 5, p. 463-494, 2006.

COELHO, R. L. On the concept of force: how understanding its history can improve physics teaching. Science \& Education, Dordrecht, v. 19, n. 1, p. 91, 2010. DOI: https:// doi.org/10.1007/s11191-008-9183-1.

COLL, R. K.; FRANCE, B.; TAYLOR, I. The role of models/and analogies in science education: implications from research. International Journal of Science Education, Abingdon, v. 27, n. 2, p. 183-198, 2005.

CUSTÓDIO, J. F.; PIETROCOLA, M. Princípios nas ciências empíricas e o seu tratamento em livros didáticos. Ciência \& Educação, Bauru, v. 10, n. 3, p. 383-399, 2004. DOI: https://doi.org/10.1590/S1516-73132004000300006.

DUIT, R.; TREAGUST, D. F. Conceptual change: a powerful framework for improving science teaching and learning. International Journal of Science Education, Abingdon, v. 25, n. 6, p. 671-688, 2003.

FODOR, J. A. The modularity of mind: an essay on faculty psychology. Cambridge: MIT Press, 1983.

FRANCO, C.; COLINVAUX, D.; KRAPAS, S.; QUEIROZ, G. A teoria piagetiana e os modelos mentais. In: BANKS-LEITE, L. (org.). Percursos piagetianos. São Paulo: Cortez, 1997. p. 187-206.

GILBERT, J. K.; PIETROCOLA, M.; ZYLBERSZTAJN, A.; FRANCO, C. Science and education: notions of reality, theory and model. In: GILBERT, J. K.; BOULTER, C. Developing models in science education. Dordrecht: Springer, 2000. p. 19-40.

GILBERT, J. K.; JUSTI, R. Modelling-based teaching in science education. Dordrecht: Springer, 2016.

GRECA, I. M.; MOREIRA, M. A. Mental models, conceptual models, and modelling. International Journal of Science Education, Abingdon, v. 22, n. 1, p. 1-11, 2000.

HARRISON, A. G.; TREAGUST, D. F. Modelling in science lessons: are there better ways to learn with models? School Science and Mathematics, Hoboken, v. 98, n. 8, p. 420-429, 1998.

HARRISON, A. G.; TREAGUST, D. F. A typology of school science models. International Journal of Science Education, Abingdon, v. 22, n. 9, p. 1011-1026, 2000.

JAMMER, M. Concepts of mass in contemporary physics and philosophy. Princeton: Princeton University Press, 2000.

JOHNSON-LAIRD, P. N. Mental models: towards a cognitive science of language, inference, and consciousness. Cambridge: Harvard University Press, 1983. 
KARAM, R. A. S.; PIETROCOLA, M. Habilidades técnicas versus habilidades estruturantes: resolução de problemas e o papel da matemática como estruturante do pensamento físico.

Alexandria, Florianópolis, v. 2, n. 2, p. 181-205, 2009.

KARMILOFF-SMITH, A.; INHELDER, B. If you want to get ahead, get a theory.

Cognition, Amsterdam, v. 3, n. 3, p. 195-212, 1975.

KARMILOFF-SMITH, A. Beyond modularity: a developmental perspective on cognitive science. Cambridge: MIT Press, 1992.

LIMÓN, M. On the cognitive conflict as an instructional strategy for conceptual change: a critical appraisal. Learning and Instruction, Kidlington, v. 11, n. 4, p. 357-380, 2001.

MACHADO, J.; BRAGA, M. Secondary students' modelling conceptualisation in situations related to particle dynamics: a clinical perspective. International Journal of Science Education, Abingdon, v. 40, n. 13, p. 1-23, 2018. DOI: https://doi.org/10.1080/09500693. 2018.1494394.

MATTHEWS, M. R. Models in science and in science education: an introduction. Science $\boldsymbol{\&}$ Education, Dordrecht, v. 16, n. 7-8, p. 647-652, 2007.

MESSER, D. J.; PINE, K. J.; BUTLER, C. Children's behaviour and cognitions across different balance tasks. Learning and Instruction, Kidlington, v. 18, n. 1, p. 42-53, 2008. DOI: https://doi.org/10.1016/j.learninstruc.2006.09.008.

MOREIRA, M. A. Modelos mentais. Investigações em Ensino de Ciências, Porto Alegre, v. 1, n. 3, p. 193-232, 1996.

MORTIMER, E. F. Construtivismo, mudança conceitual e ensino de ciências: para onde vamos? Investigações em Ensino de Ciências, Porto Alegre, v. 1, n. 1, p. 20-39, 1996.

NARDI, R.; GATTI, S. R. T. Uma revisão sobre as investigações construtivistas nas últimas décadas: concepções espontâneas, mudança conceitual e ensino de ciências. Ensaio, Belo Horizonte, v. 6, n. 2, p. 129-150, 2008. DOI: https://doi.org/10.1590/198321172004060205.

NERSESSIAN, N. J. The cognitive basis of model-based reasoning in science. In: CARRUTHERS, P.; STICH, S.; SIEGAL, M. (ed.). The cognitive basis of science. Cambridge: Cambridge University Press, 2002. p. 133-153.

NERSESSIAN, N. Constructing and instructing: the role of "abstraction techniques" in creating and learning physics. In: DUSCHL, R. A.; HAMILTON, R. J. (org.). Philosophy of science, cognitive psychology, and educational theory and practice. New York: Suny Press, 1992. p. 48-68.

NERSESSIAN, N. J. Should physicists preach what they practice? In: BERNARDINI, C.; TARSITANI, C.; VICENTINI, M. (ed.). Thinking physics for teaching. New York: Springer, 1995. p. 77-96.

PIAGET, J. Epistemologia genética. 4. ed. São Paulo: WMF Martins Fontes, 2012. 
PIETROCOLA, M. A matemática como estruturante do conhecimento físico. Caderno Brasileiro de Ensino de Física, Florianópolis, v. 19, n. 1, p. 93-114, 2002.

PINE, K.; MESSER, D. The development of representations as children learn about balancing. British Journal of Developmental Psychology, Oxford, v. 21, n. 2, p. 285-301, 2003.

PINE, K. J.; MESSER, D. J. Group collaboration effects and the explicitness of children's knowledge. Cognitive Development, London, v. 13, n. 1, p. 109-126, 1998.

PINE, K.; MESSER, D. J. What children do and what children know: looking beyond success using Karmiloff-Smith's RR framework. New Ideas in Psychology, Kidlington, v. 17, n. 1, p. 17-30, 1999.

POSNER, G. J.; STRIKE, K. A.; HESON, P. W.; GERTZOG, W. A. Accomodations of a scientific conception: toward a theory of conceptual change. Science Education, Hoboken, v. 66, n. 22, p. 211-227, 1982.

POZO, J. I.; GÓMEZ CRESPO, M. A. The embodied nature of implicit theories: The consistency of ideas about the nature of matter. Cognition and Instruction, Philadelphia, v. 23, n. 3, p. 351-387, 2005.

SHTULMAN, A.; VALCARCEL, J. Scientific knowledge suppresses but does not supplant earlier intuitions. Cognition, Amsterdam, v. 124, n. 2, p. 209-215, 2012.

SIEGLER, R. S. Three aspects of cognitive development. Cognitive Psychology, Maryland Heights, v. 8, n. 4, p. 481-520, 1976.

TABER, K. S. The natures of scientific thinking: creativity as the handmaiden to logic in the development of public and personal knowledge. In: KHINE, M. S. (ed). Advances in nature of science research. Dordrecht: Springer, 2012. p. 51-74.

VERGNAUD, G. Pourquoi la théorie des champs conceptuels? Infancia y Aprendizaje, Madrid, v. 36, n. 2, p. 131-161, 2013.

VERGNAUD, G. Psychologie du développement cognitif et didactique des mathématiques. Grand N, Grenoble, v. 38, p. 21-40, 1986.

VOSNIADOU, S.; BREWER, W. F. Mental models of the earth: A study of conceptual change in childhood. Cognitive Psychology, Maryland Heights, v. 24, n. 4, p. 535-585, 1992.

Submetido em: 7/11/2018. Aceito em: 10/01/2019

Contato: CEFET, Av. Maracanã, 229, Rio

de Janeiro, RJ 20271-110, Brasil. 